\title{
Gene Mutation Characteristics and Cluster Analysis of Multidrug-Resistant Mycobacterium Tuberculosis in Urumqi
}

Jiandong Yang ( $\sim$ YJD3631@163.com )

Urumqi Centers for Disease Control and Prevention

Rayibai Yadikaer

Health and Family Planning Commission of Xinjiang Uygur Autonomous Region

Yanggui Chen

Urumqi Center For Disease Control and Prevention

Li Ma

Urumqi Centers for Disease Control and Prevention

Yaoqin Lu

Urumqi Centers for Disease Control and Prevention

Na Xue

Urumqi Centers for Disease Control and Prevention

Baolin Rui

Urumqi Centers for Disease Control and Prevention

Research

Keywords: Tuberculosis, Drug-resistant tuberculosis, Molecular epidemiological characteristics

Posted Date: January 16th, 2021

DOI: https://doi.org/10.21203/rs.3.rs-144626/v1

License: (c) (i) This work is licensed under a Creative Commons Attribution 4.0 International License. Read Full License 


\section{Abstract}

Objective To understand the molecular biological characteristics of multidrug-resistant Mycobacterium tuberculosis in Urumqi by the analysis of gene mutation and clustering of multidrug-resistant Mycobacterium tuberculosis in Urumqi, to provid theoretical basis for the prevention and control of multidrug-resistant tuberculosis in the future.

Methods The tuberculosis patients who were diagnosed, registered, cultured positive Mycobacterium tuberculosis and over 16 years old in Urumqi were studied. Geno Type MTBDR plus Assay and MIRU-VNTR were used to analysis the gene mutation and clustering of multidrug-resistant mycobacterium tuberculosis in Urumqi.

Result Mutation gene included Isoniazid resistance gene katG 315; Rifampicin resistance gene rpoB 526, 531; Ethambutol resistance gene embB 206; Streptomycin resistance gene rpsL 43 and RRs 1401; Ofloxacin resistance gene gyrA 74, 90, 91 and 94. It was shown that the Beijing genotype was predominant (49.530\%) among these319strains. There were 14 strains multi-drug resistant strains, it was predominant (73.684\%) of multi-drug resistant strains. Multi-drug resistant prevalence of Beijing strains was $8.861 \%$. The clustering rate of MDR-TB was $5.263 \%$ in Urumqi.

Conclusion Molecular biological characteristics of multidrug-resistant Mycobacterium tuberculosis have some particularities in Urumqi. Therefore, modern molecular epidemiological techniques such as molecular biology and DNA fingerprinting can be used to improve the speed of diagnosis and to carry out case tracking and source investigation.

\section{Introduction}

Tuberculosis (TB) is a chronic infectious disease and it is called "white plague" with the largest number of human deaths [1]. At the same time, it is still the main killer endangering human life. According to the Global Tuberculosis Report of 2018, it is estimated that in 2017 there will be about 10 million new TB cases worldwide, the incidence of TB will be $133 / 100,000$, the global estimated number of TB deaths will be about 157,000 , and the mortality rate will be 17/100,000. China is one of the 22 countries who with high TB burden in the world, it is the third just followed India and Indonesia in the word. In recent years, the emergence of drug resistance tuberculosis (DR-TB) worldwide, especially multi-drug resistant tuberculosis (MDR-TB) posed a serious challenge to global TB prevention and control. The Word Health Organization (WHO) estimated that $3.6 \%$ of new patients and $17 \%$ of retreated patients worldwide in 2017 will be multi-drug resistant tuberculosis or rifampicin resistant tuberculosis (MDR/RR-TB) patients ${ }^{\text {[2] }}$.

China was one of the most serious disaster areas of MDR-TB. The rate of MDR-TB has been increasing in recent years ${ }^{[3]}$. The results of the fifth national tuberculosis epidemiological sampling survey in 2010 showed that: The total multi-drug resistance rate is $6.8 \%$. The initial multi-drug resistance rate is $5.4 \%$. The acquired multi-drug resistance rate is $15.4 \%$ [4]. Studies showed that there are significant differences in the distribution of MDR-TB in different regions ${ }^{[5]}$. Xinjiang as one of serious TB disaster area in China, the epidemic situation of MDR-TB is also not optimistic. The results of the fifth tuberculosis epidemiological sampling survey in Xinjiang Uygur Autonomous Region showed that the total multi-drug resistance rate was $1.16 \%{ }^{[6]}$.Although lower than the national level, but from the incidence trend was more serious ${ }^{[7] . F r o m ~ t h i s ~ v i e w, ~ M D R-T B ~ h a s ~ b e c o m e ~ a ~ m a j o r ~ p u b l i c ~ h e a l t h ~ p r o b l e m ~ i n ~ C h i n a ~ a n d ~ e v e n ~ i n ~ t h e ~ w o r l d, ~ t h a t ~ n e e d ~ a t t a c h ~}$ great attention .

With the increasing of MDR-TB global epidemic, the research on the influencing factors of MDR-TB was also deepening studied by many countries. Studies showed that the incidence of MDR-TB is related to gene mutation of Mycobacterium tuberculosis (MTB), socio-economic factors, living standards, living conditions, regional health resources allocation and service quality, meteorological factors, geographical factors, government support, implementation of TB strategy and TB management. Gene mutation is related to social and ecological factors, which is the result of the interaction of many factors in recent years ${ }^{\left[{ }^{-11}\right.}$, researchers focused on basic bacteriological and molecular epidemiological studies of MTB. 
The results of foreign studies showed that Beijing family type was the most dominant genotype of multi-drug resistant MTB ${ }^{[12]}$. Compared to other types, Beijing family type was more likely to cause MDR-TB in patients worldwide. The second was $\mathrm{H} 3$ type. It's risk of MDR-TB was only lower than Beijing type, but significantly higher than the other TB types

[13]. The results were similar to foreign studies. Beijing type was the main pathogenic type ${ }^{[14-15]}$. Some scholars have suggested that this may be related to the virulence and infectivity of MTB strains with Beijing family genotype ${ }^{[12]}$. In addition to the influence of strain genotype on the pathogenesis of MDR-TB, mutation of drug resistance gene locus of MTB is one of the most important factors lead drug resistance. Recent studies have shown that the common resistance genes of isoniazid were katG and inhA, rifampicin was rpoB, ethambutol was embB, streptomycin was rpsL and ofloxacin was gyrA. Among them, katG, inhA and rpoB were the most frequent in MDR-TB patients ${ }^{[16-17]}$. The highest mutation rate of isoniazid resistance gene katG was 315 sites, and the highest mutation rate of inhA gene was 15 sites. But the highest mutation site in rpoB of Rifampicin was 531, 526 and 516. At the same time, some researchers said that there may have joint mutations between drug-resistant genes ${ }^{[18-20]}$. In addition, molecular epidemiology is also expanding the scope of research, such as gene clustering analysis in the study of strain homology, DNA fingerprinting technology in the MDR-TB outbreak, epidemiological investigation and so on.

In this study, molecular epidemiological methods were used to understand the molecular epidemiological characteristics of MTB and its impact on the incidence of MDR-TB in the market.

\section{Material And Methods}

\section{Mycobacterium tuberculosis isolates}

The survey was cStandardsonducted in eight tuberculosis designated hospitals in the city from January 2014 to March 2015. The eight designated hospitals provide services to all TB patients in Urumqi. 319 tuberculosis patients were enrolled in this study. In addition, sputum samples were collected from patients at different time points (point, morning and evening). Acid-fast staining and Roche culture were used for microscopic examination and isolation of MTB.

\section{Drug susceptibility testing}

All MTB isolates were tested by proportional method on Roche susceptibility medium (DST). The drug concentration was isoniazid $(0.2 \mathrm{~g} / \mathrm{ml})$, Li Fuping $(40 \mathrm{~g} / \mathrm{ml})$, streptomycin $(4 \mathrm{~g} / \mathrm{ml})$, ethambutol $(2 \mathrm{~g} / \mathrm{ml})$, ofloxacin $(2 \mathrm{~g} / \mathrm{ml})$, kanamycin $(30 \mathrm{~g} / \mathrm{ml}),(40(\mathrm{~g} / \mathrm{ml})$ and curreomycin $(40 \mathrm{~g} / \mathrm{ml})$. The identification of MTB strains was carried out by using the identification medium of MTB.

\section{DNA extraction and sequencing}

The primers used in this study were obtained from refer to literatures. The primers were synthesized by "Hua Da Gene". DNA of MTB was extracted by ultrasonic lysis. Specific methods as follows: $500 \mathrm{ml}$ sputum specimens added into $1.5 \mathrm{ml}$ centrifugal tube; centrifugal for 5 minutes at $12000 \mathrm{r} / \mathrm{min}$, discarding supernatant, adding $500 \mathrm{ml}$ sterilized water, swirling suspension precipitate, 12 000r/min centrifugation for 5 minutes, discarding supernatant, adding $100 \mathrm{ml}$ sterilized water to each tube, swirling suspension; incubation specimens at $95^{\circ} \mathrm{C}$ for 20 minutes in water bath, ultrasonic decomposition for 15 minutes, and $12000 \mathrm{r} /$ centrifugation for 5 minutes. Minutes later, the supernatant was transferred to a new $500 \mathrm{ml}$ centrifugal tube and stored at $-20^{\circ} \mathrm{C}$ for reserve. The extracted DNA was amplified. Finally, the PCR products were purified and sequenced on 3730 DNA analyzer.

\section{Genotyping method}

MIRU02, MIRU04, MIRU10, MIRU16, MIRU20, MIRU23, MIRU24, MIRU26, MIRU27, MIRU31, MIRU39 and MIRU40 were selected according to the bacterial gene database. There were 12 tandem repeat loci. The amplified product of $5 \mu$ PCR 
was added with $1 \mu \mathrm{l}$ sample buffer, placed in the sample pore, covered with electrophoresis cell and connected with power supply. The electrophoresis was carried out by $1-5 \mathrm{v} / \mathrm{cm}$ voltage drop (calculated by the distance between the two poles). Finally, the ultraviolet gel imaging system was used for image preservation.

\section{Analysis method}

The data were analyzed by $\chi^{2}$ test, Fisher exact probability test and cluster analysis. The software used Stata 14.0 package and cluster analysis online MIRU-VNTRplus statistical analysis tool(http://www.miru-vntrplus.org).

\section{Standards}

\section{Diagnostic criteria for MDR-TB}

Diagnostic criteria of tuberculosis cases refer to diagnostic criteria for health industry standard of People's Republic of China (WS288-2017).

\section{Inclusion criteria}

(1) Registered by the municipal tuberculosis prevention and control agency in Urumqi;

(2) Diagnosis as TB cases refer to diagnostic criteria for health industry standard of People's Republic of China (WS2882017).

(3) TB patients with initial treatment or retreatment;

(4) MTB strains;

(5) Mycobacterium positive.

\section{Exclusion criteria}

(1) Whose age under 16 years;

(2) Who don't agree to participate in this survey;

(3) Who with severe complications.

\section{Standards of quality control}

(1) Strictly abide by the Laboratory Biosafety Manual to ensure participants safety.

(2) Testing should be strict accordance with the test operation steps stipulated of the national or industrial standards to avoid measurement bias caused by different test conditions and procedures.

(3) The reagents and safety protective devices used in the test were all in effective use, otherwise will be discarded. All the equipment and instruments required in the test shall be affixed with the qualified green label of the national quality inspection department and the metrology department, and within the validity period, the systematic bias caused by the instrument and equipment shall be avoided.

\section{Results}

\section{Distribution of mutation drug resistance genes}

Resistance genes and locus of strains that multidrug-resistant MTB was tested. The result show that only katG gene is mutation in resistance genes of isoniazid (INH), and the mutation have significance compared with the group of sensitive to all drug (Pख0.05). The mutation locus of resistance gene katG is 315 locus, and base pair AGC has changed to AAC or 
ACC, and amino acid serine (Ser) has changed to asparagine (Asn) or threonine (Thr). Resistance gene ropB of rifampicin (RFP) has mutation, and the mutation locus are 526 and 531. The base pair CAC of locus 526 and base pair TCG of locus 531 have changed to TAC and TTG respectively, and corresponding histidine (His) and serine (Ser) has changed to tyrosine (Tyr) and Leucine (Leu) respectively. It is significance compared with group of sensitive to all drug (P凶0.05). In aspect of ethambutol (EMB), resistance gene embB has mutation, and the mutation locus is 206. The base pair ATG has changed to ATA and GTG. However, compared with group of sensitive to all drug, base pair ATG changed to GTG is not significance $(P=0.119)$. In aspect of streptomycin $(S M)$, resistance gene rpsL and rrs have mutation, and the mutation locus are 43 and 1401 respectively, and base pair AGG and A have changed to AAG and G respectively. Moreover, corresponding Arginine (Arg) and alanine (Ala) have changed to lysine (Lys) and glycine (Gly) respectively. Compared with group of sensitive to all drug, these mutation are significant difference (P凶0.05). The mutation locus of resistance gene gyrA in ofloxacin (OFX) have four. These locus are 74,90,91 and 94 . With the exception of 90 locus, the mutation of other locus have both significant difference (P凶0.05). In addition, we found also that there is not mutation of resistance gene in capreomycin (CMP), kanamycin (KAM) and amikacin (AMK). (see Table 1)

Table 1

The mutation characteristics of resistance gene in multi-drug resistant MTB

\begin{tabular}{|c|c|c|c|c|c|c|c|c|c|}
\hline \multirow[t]{2}{*}{ Drugs } & \multirow[t]{2}{*}{ Gene } & \multirow[t]{2}{*}{ Locus } & \multirow[t]{2}{*}{ Mutation } & \multirow{2}{*}{$\begin{array}{l}\text { Amino } \\
\text { acid }\end{array}$} & \multicolumn{2}{|c|}{ Multi drug $(n=19)$} & \multicolumn{2}{|c|}{ All sensitive $(n=254)$} & \multirow[t]{2}{*}{$P^{*}$} \\
\hline & & & & & $\begin{array}{l}\text { Mutant } \\
\text { strains(n) }\end{array}$ & $\begin{array}{l}\text { Mutation } \\
\text { rates(\%) }\end{array}$ & $\begin{array}{l}\text { Mutant } \\
\text { strains(n) }\end{array}$ & $\begin{array}{l}\text { Mutation } \\
\text { rates(\%) }\end{array}$ & \\
\hline \multicolumn{10}{|l|}{$\mathrm{INH}$} \\
\hline & katG & 315 & $\mathrm{AGC} \rightarrow \mathrm{AAC}$ & Ser $\rightarrow$ Asn & 3 & 15.789 & 0 & 0.000 & $\varangle 0.001$ \\
\hline & katG & 315 & $\mathrm{AGC} \rightarrow \mathrm{ACC}$ & $\mathrm{Ser} \rightarrow \mathrm{Thr}$ & 14 & 73.684 & 0 & 0.000 & $\varangle 0.001$ \\
\hline & $\operatorname{inh} A$ & - & - & - & 0 & 0.000 & 0 & 0.000 & - \\
\hline \multicolumn{10}{|l|}{ RFP } \\
\hline & гров & 526 & $\mathrm{CAC} \rightarrow \mathrm{TAC}$ & His $\rightarrow$ Tyr & 2 & 10.526 & 0 & 0.000 & $\nabla 0.001$ \\
\hline & гров & 531 & $\mathrm{TCG} \rightarrow \mathrm{TTG}$ & Ser $\rightarrow$ Leu & 14 & 73.684 & 0 & 0.000 & $₫ 0.001$ \\
\hline \multicolumn{10}{|l|}{ EMB } \\
\hline & embB & 206 & ATG $\rightarrow$ ATA & Met $\rightarrow$ Ile & 4 & 21.053 & 11 & 4.331 & 0.014 \\
\hline & embB & 206 & $\mathrm{ATG} \rightarrow \mathrm{GTG}$ & Met $\rightarrow$ Val & 3 & 15.789 & 15 & 5.906 & 0.119 \\
\hline \multicolumn{10}{|l|}{ SM } \\
\hline & rpsL & 43 & $\mathrm{AGG} \rightarrow \mathrm{AAG}$ & Arg $\rightarrow$ Lys & 10 & 52.632 & 2 & 0.787 & $\otimes 0.001$ \\
\hline & rrs & 1401 & $A \rightarrow G$ & Ala $\rightarrow$ Gly & 3 & 15.789 & 2 & 0.787 & 0.003 \\
\hline \multicolumn{10}{|l|}{ OFX } \\
\hline & gyrA & 74 & $\mathrm{GCC} \rightarrow \mathrm{TCC}$ & $\mathrm{Ala} \rightarrow \mathrm{Ser}$ & 1 & 5.260 & 0 & 0.000 & 0.070 \\
\hline & gyrA & 90 & $\mathrm{GTC} \rightarrow \mathrm{GTG}$ & $\mathrm{Val} \rightarrow \mathrm{Val}$ & 1 & 5.260 & 1 & 3.393 & 0.135 \\
\hline & gyrA & 91 & $\mathrm{TCG} \rightarrow \mathrm{CCG}$ & Ser $\rightarrow$ Pro & 2 & 10.526 & 0 & 0.000 & 0.005 \\
\hline & gyrA & 94 & $\mathrm{GAC} \rightarrow \mathrm{AAC}$ & Asp $\rightarrow$ Asn & 3 & 15.789 & 0 & 0.000 & $\varangle 0.001$ \\
\hline & gyrA & 94 & $\mathrm{GAC} \rightarrow \mathrm{GGC}$ & Asp $\rightarrow$ Gly & 1 & 5.260 & 0 & 0.000 & 0.070 \\
\hline
\end{tabular}

\section{The distribution characteristic of gene type}


We had genotyping to multi-resistance drug MTB strains through method of 12 locus MIRU-VNTR gene type. The result show that the kind of gene type is four that Beijing type, T1 type, T2 type and Special type in multi-drug resistance MTB. And most of multi-drug resistance rate is Beijing type (8.861\%), the next of Special type (7.500\%), the last is T1 type (1.282\%). (see Table 2)

Table 2

Genotype distribution of multi-drug resistant MTB

\begin{tabular}{|llll|}
\hline Gene type & Number $(\boldsymbol{n})$ & Number of multi-drug $(\boldsymbol{n})$ & Rates of multi drug resistant MTB (\%) \\
\hline Beijing type & 158 & 14 & 8.861 \\
\hline T1 type & 78 & 1 & 1.282 \\
\hline T2 type & 21 & 1 & 4.762 \\
\hline Special type & 40 & 3 & 7.500 \\
\hline
\end{tabular}

\section{Cluster analysis}

After genotyping, we had cluster analysis on the basis of genotyping. We find that number of clusters is one, if we believe similarity above $95 \%$ is one cluster (see Fig. 1). According to conclusions from above, if we assume that one strain in each cluster is infection source of the cluster, then we estimate that $5.263 \%$ [( $2-1 / 19]$ of peoples who infect multi-drug resistant MTB are because of infected. The result show, when maximum complex trajectory difference equal to 2 (DL $=2$ ), the optimal propagation path fitting of significance in multi drug resistant MTB is three group, which it is precondition that without consider other factor and only consider relationship between genes of multi drug resistant MTB. The three

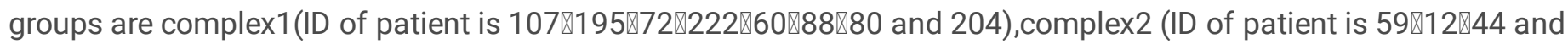
53), and complex3(ID of patient is 6 and 58). It is interesting that gene type is absolutely identical and the similarity distance is zero between patient 107 and patient 195. (see Fig. 1 and Fig. 2)

\section{Discussion}

At present, mutation of the first-line anti-tuberculosis drug resistance genes have been extensively studied at home and abroad, such as INH, RFP, EMB and SM. Studies showed that the mutation sites of the drug resistance genes of the above anti-tuberculosis drugs are quite different in different regions ${ }^{[21]}$.This study showed that the mutation rates of isoniazid resistance gene katG, rifampicin resistance gene rpoB, ethambutol resistance gene embB, streptomycin resistance gene $r p s L$ and RRs in multidrug resistance group were higher than those in full-sensitivity group, which consistent with the results of domestic or foreign research ${ }^{[22-25]}$. However, there was no mutation in inhA gene of isoniazid-resistant group and full-sensitive group, which was inconsistent with the results of Asho Ali et al. The results showed that the mutation rate of inhA gene was about $8 \%{ }^{[26]}$. This difference may relate with geographical differences. At the same time, the results showed that the mutation site of INH resistance gene katG was 315. The base pairs of INH resistance gene katG were converted from AGC to AAC and AGC to ACC, while the amino acids were converted from Ser to Asn and Thr, this result consistent with Mekonnen D et al ${ }^{[27]}$. Studies showed that the anti-tuberculosis mechanism of INH is mainly to inhibit the synthesis of cell wall of MTB and make it lose its proliferative ability, which leads to the apoptosis of MTB [28]. In this process, the KatG gene of MTB (catalase peroxidase encoding gene) plays a key role. The above-mentioned antituberculosis effect of INH could be accomplished only by converting it into its biological active isonicotinic acid under the action of catalase-peroxidase. The codon mutation at the 315 locus of katG gene resulted in the conversion of amino acids from serine to asparagine (Asn) and threonine (Thr), caused a change in the protein structure encoded by the katG gene, leaded a decrease in catalase peroxidase activity, which ultimately prevented the conversion of isoniazid to its bioactive isoniazid, resulted in the loss of isoniazid resistance ${ }^{[29]}$. Because there was no inhA gene mutation in this study, there was no combined mutation of katG gene and inhA gene, which resulted in isoniazid resistance in MDR-MTB. This 
could provide some reference to future genetic diagnosis of MDR-TB in Urumqi. The highest mutation rate of RFP resistance gene rpoB was 531. The base of RFP was transformed from TCG to TTG, and the amino acid was transformed from Ser to Leu. This was consistent with the results of Nwofor AC and Hu Qiong et al ${ }^{[30]}$. In terms of mechanism, serine at the 531 site of rpoB gene was the most critical target for RFP to act on MTB. Once mutation occurs, it will directly lead RFP resistance in MTB. At the same time, the mutation at 526 site of rpoB gene also occurred in this study, suggesting that there may be a joint mutation at 526 site and 531 site. The embB mutation of the EMB resistance gene was 206; the base group was mainly converted from ATG to ATA, and the amino acid was converted from Met to lle. This was inconsistent with Khosravi AD et al.'s research, which showed that 306 site is a common mutation site of embB of the EMB resistance gene ${ }^{[31]}$.It was suggested that the embB gene of multi-drug resistant MTB may have some regional specificity, which can be used as a reference site for gene screening and rapid diagnosis of drug-resistant TB in Urumqi. The results are consistent with those of Smittipat N, Rezaei F and others ${ }^{[32-33]}$. The results showed that the mutation sites of rpsL and RRs of SM resistance gene were 43 and 1401 ; the mutation bases of 43 sites were transformed from AGG to AAG, and the mutation base pairs of 1401 sites were transformed from A to $G$. The mutations of rpsL and RRs resulted in the encoding disorders of the glycosomal protein S12 and ribosomal 16SrRNA, respectively, leading to the

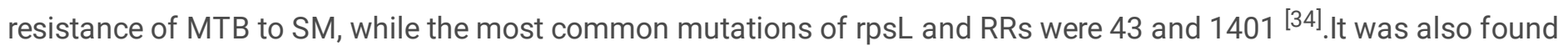
that there were joint mutations in the SM resistance genes rpsL and rrs. Mutation analysis of drug resistance genes of second-line drugs showed that only gyrA gene of OFX resistance mutated in four second-line anti-tuberculosis drugs, and the mutation sites were 74, 90, 91 and 94 . This is consistent with the results of Wang Z, Sirous M and others ${ }^{[35-36]}$.From the above analysis of drug resistance gene site we could see that there were not only the genetic and molecular biological characteristics of the mainstream MDR-TB strains at home and abroad, but also has a few special characteristics. These characteristics will provide a new idea for rapid detection of MDR-TB in Urumqi in the future. With the deepening of molecular biology research on multi-drug resistant MTB strains, it was also suggested that the specialized TB prevention and control institutions and specialized hospitals in Urumqi should make full use of modern MDR-TB diagnosis technology to make rapid and accurate diagnosis, so as to gain valuable time for follow-up treatment. According to the genotype distribution characteristics of MTB: In this study, 12-locus MIRU-VNTR genotyping method was used to genotype multidrug-resistant MTB in Urumqi. It was found that 19 strains of multidrug-resistant MTB could be divided into four genotypes, named Beijing type, T1, T2 and idiotype. Among them, the best potential genotype of multidrugresistant MTB in Urumqi was Beijing type. This result consistent with Yuan $L$ et al ${ }^{[37]}$. MTB with Beijing genotype has the characteristics of rapid transmission, strong virulence and wide epidemic range. According to the results of genotyping, the dominant strain of MDR-TB in Urumqi is Beijing, which give a new challenge to the prevention and control of MDR-TB in Urumqi. It also suggests that the disease prevention and control agencies should pay close attention to monitoring drug resistance (especially RNH and RFP) in the treatment and management of TB patients whose strain genotype is Beijing in the future, and adjust the treatment plan in time to avoid the deterioration of the disease and the multi-drug resistance of MTB. This study also found that the multidrug resistance rate of the idiotype was second just behind Beijing type. This phenomenon may be caused by the large floating population and frequent population flow in our city, leaded a wide range of genotypes of MTB strains, and the idiotype produced by genotype-related hybridization of each strain. This idiotype retains the predominant resistance characteristics of the original genotypes, which make it easier for the idiotype to produce multi-drug resistance. In terms of clustering, MIRU-VNTR technique at 12 locus was used to cluster 19 strains of MTB isolated from multidrug-resistant patients. The results showed that 2 of 19 MDR-TB patients could be clustered into one cluster. It was inferred that the homology of MDR-TB in Urumqi is $5.263 \%$, which is slightly lower than similar studies ${ }^{[38]}$. This may be related to the implementation of the new Trinity TB management model in our city since 2012. The Trinity TB management model refers to the establishment of a clear division of labor and coordinated service system for TB prevention and control agencies, designated medical institutions and grass-roots medical and health institutions. The construction of this prevention and control system greatly improved the efficiency of TB from prevention, diagnosis, treatment to management, and could discover the source of infection in time, control it at the same time, effectively blocking the spread of tuberculosis. Under this model, the incidence of MDR-TB and the probability of homologous

Page $7 / 13$ 
transmission of MDR-TB could be reduced ${ }^{[39]}$. As an effective modern TB management mode, the Trinity management mode should be implemented by the disease prevention and control agencies unremittingly. On this basis, combined with Urumqi TB epidemic situation, regional characteristics and other aspects of improvement, in order to adapt the actual needs of TB prevention and control work, greatly meet and ensure the actual interests of TB patients. Further analysis of the minimum spanning tree under complex paths showed that there were 3 optimal transmission paths for 19 MDR-TB patients. At the same time, combined with the results of genotyping, it was found that there might be a relationship between infection and infection between patients 107 and patients 195. By reviewing the questionnaires of patients No. 107 and No. 195, easy to find that they did not live in the same district and county, but the occupations of patients No. 107 and No. 195 were worker and farmers, which suggested that there may be employment relationship between them. The field epidemiological case study found that there was no social relationship between them, such as relatives, friends and colleagues, but there was employment relationship between them. At the same time, we know that No. 195 earlier infection TB at December 10, 2013, and the confirmation time was September 10, 2014. First time of No. 107 infection TB was September 2, 2014, and the confirmation time was September 19, 2014. Meanwhile, patients 107 worked in villages where patients No. 195 lived from January 2012 to September 2014, and patients No. 195 employed patients 107 during 2014. They had close contact with each other, mainly in farmland and the residence of patients No. 195. Two people recalled that the contact time between the two people in the residence of patient No. 195 was longer than that in the farmland, but the specific contact time could not be confirmed. We believe that patients 107 are more likely to be infected in the residence of patients 195.The preliminary success of the Etiology Survey provides relevant experience for the future investigation of TB outbreaks, especially MDR-TB outbreaks in our city. According to the latest reports, breakthroughs have been made in the application of molecular epidemiological methods such as gene fingerprinting, gene homology and cluster analysis in source tracking and source identification, and they have been gradually applied in practice, especially in source identification and chain tracking of major infectious disease outbreaks ${ }^{[40-41]}$. Moreover, this method had high specificity, high efficiency and high speed, which had won valuable time for rapid eradication of the epidemic situation ${ }^{[42]}$. Therefore, molecular epidemiological techniques should be further developed in the investigation of TB outbreaks in order to achieve scientific and rapid prevention and control.

At present, the study on molecular epidemic characteristics of multidrug-resistant MTB in Urumqi city level is still blank. This was the first report on the molecular biology of MDR-TB in Urumqi. At the same time, this study provided relevant experience for the future prevention and control of MDR-TB and outbreak investigation (mainly referring to the tracing of infectious sources) in Urumqi, and also provided reference for the research to other related infectious diseases. In addition, the results of this study will have great scientific value and social and economic effects on the prevention and control of MDR-TB in Urumqi. The study was influenced by time, long treatment cycle and low incidence of MDR-TB. In fact, only 319 eligible patients were surveyed in limited research time. Only 19 cases of MDR-TB were found. Although the sample size can basically meet the needs of analysis, the sample size was still insufficient. In the future, the investigation time can be prolonged appropriately, more MDR-TB cases can be collected, and more accurate data can be obtained. Secondly, although the results of this study were the same as those of the mainstream research at home and abroad, they also have their own characteristics. Because the research in this field in Urumqi is still blank, it could not provide evidence for this study. Therefore, further studies are needed to provide evidence for these specific results.

\section{Conclusion}

INH resistance gene katG315 mutation, RFP resistance gene rpoB526, 531 mutation, EMB resistance gene embB206 mutation, SM resistance gene rpsL, RRs corresponding 43, 1401 mutation and OFX resistance gene gyrA74, 90, 91 and 94 mutation; 12 MIRU-VNTR site genetic differences and typing. Differentiation was characterized by small genetic differences and high typing discrimination $(\mathrm{HGI}=0.725)$; genotype distribution was characterized by Beijing type with the 
highest rate of multi-drug resistance (8.861\%); cluster analysis showed that the clustering rate of MDR-TB in Urumqi was $5.263 \%$.

\section{Declarations}

\section{Acknowledgements}

We acknowledge the Urumqi Education Department and Urumqi Center for Disease Control and Prevention for giving support to conduct the study.

\section{Funding}

This work was supported by Health Youth Medical Science and Technology Talents Special Research Project of Xinjiang Uygur Autonomous Region, China [WJWY202109] and Natural Science Foundation of Xinjiang Uygur Autonomous Region, China [201442137-20].

\section{Ethics approval and consent to participate}

Not applicable.

\section{Competing interests}

The authors declare that they have no competing interests.

\section{Author details}

Yang Jiandong (Department for Tuberculosis Control and Prevention, Urumqi Center for Disease Control and Prevention, Urumqi, Xiamen Road 18, 830026, Xinjiang, China. E-mail:YJD3631@163.com); Rayibai-Yadikaer(Health inspection of Health and Family Planning Commission of Xinjiang Uygur Autonomous Region, Urumqi, Longquan Road 191, 830001, Xinjiang, China. E-mail:420383531@qq.com); Chen Yanggui (Department for Tuberculosis Control and Prevention, Urumqi Center for Disease Control and Prevention, Urumqi, Xiamen Road 18, 830026, Xinjiang, China. E-mail:cdctb@126.com); Ma Li (Department for Tuberculosis Control and Prevention, Urumqi Center for Disease Control and Prevention, Urumqi, Xiamen Road 18, 830026, Xinjiang, China. E-mail:497976767@qq.com); Lu Yaoqin(Department of Occupational and Environmental Health, Xinjiang Medical University School of Public Health \& Science and Education Department, Urumqi Center for Disease Control and Prevention, Urumqi, Xiamen Road 18, 830026, Xinjiang, China. E-mail:35278597@qq.com); Rui Baolin(Department for Tuberculosis Control and Prevention, Urumqi Center for Disease Control and Prevention, Urumqi, Xiamen Road 18, 830026, Xinjiang, China. E-mail:1441948546@qq.com)

\section{References}

1. Dai ZhiChen X, DongLou W. LiYa. History of middle defense consumption [M]. The first edition. Beijing: people's medical publishing house; 2013.

2. WHO. Global tuberculosis report[R]. Geneva:World Health Organization, 2018.

3. Mingguan Lin,Yeteng Zhong,Zhuolin Chen. et al. High incidence of drug-resistant Mycobacterium tuberculosis in Hainan Island. China[J]Tropical Medicine International Health. 2019;24(9):1098-103.

4. Ding P, Li X, Jia Z, et al. Multidrug-resistant tuberculosis (MDR-TB) disease burden in China: a systematic review and spatio-temporal analysis[J]. BMC Infect Dis. 2017;17(1):57.

5. Haican Liu,Yuanyuan, Zhang,Zhiguang Liu, et al. Associations between Mycobacterium tuberculosis Beijing genotype and drug resistance to four first-line drugs: a survey in China[J]. Frontiers of Medicine, 2018,12(1):92-97. 
6. Jin-ming YANG,Jie'ensi-SIMAHULE,TAI Xin-rong, et al. Analysis of tuberculosis epidemiological survey conducted in 2010-2011 in Xinjiang Uygur autonomous region [J]. Chinese Journal of Antituberculosis,2013, 35(12):960-964.

7. Li Yuqing. An Investigation Beijing Genotype and Drugs Resistance of Mycobacterium tuberculosis in the Four Province (Autonomous Regions) of North China [D]. University of south China, 2017.

8. Gerard de Vries S, Tsolova LF, Anderson, et al. Health system factors influencing management of multidrug-resistant tuberculosis in four European Union countries - learning from country experiences [J]. BMC Public Health. 2017;17(1):334.

9. Francesco Di Gennaro,Damiano Pizzol,Bonifacio Cebola. et al. Social determinants of therapy failure and multi drug resistance among people with tuberculosis: A review [J].Tuberculosis (Edinb),2017103(3):44-51.

10. Ullah I, Javaid A, Tahir Z, et al. Pattern of Drug Resistance and Risk Factors Associated with Development of Drug Resistant Mycobacterium tuberculosis in Pakistan[J]. PLoS One. 2016;11(1):e147529.

11. Mohd Shariff N, Shah SA, Kamaludin F. Previous treatment, sputum-smear nonconversion, and suburban living:The risk factors of multidrug-resistant tuberculosis among Malaysians[J]. International Journal of Mycobacteriology. 2016;5(1):51-8.

12. Olha Konstantynovska M, Rekrotchuk I, Hrek,et al. Severe Clinical Outcomes of Tuberculosis in Kharkiv Region, Ukraine, Are Associated with Beijing Strains of Mycobacterium tuberculosis [J]. Pathogens,2019,8(2): E75.

13. Asrar, Elegail. Nuha Yousif Ibrahim Mohamed, Eman Osman Mohamed Nour,et al. Molecular characterization of Mycobacterium tuberculosis isolates from pulmonary tuberculosis patients in Khartoum, Sudan [J].International Journal of Mycobacteriology,2018,7(3):236-241.

14. Liu Q, Wang D, Martinez L,et al. Mycobacterium tuberculosis Beijing genotype strains and unfavorable treatment outcomes: A systematic review and meta-analysis [J]. Clin Microbiol Infect. 2019;20(1):1198-743. X(19)30405-7.

15. Hu Y, Zhao Q, Werngren J, et al. Drug resistance characteristics and cluster analysis of M. tuberculosis in Chinese patients with multiple episodes of anti-tuberculosis treatment[J]. BMC Infect Dis. 2016;16(1):4.

16. Feriel Bouziane,Rachida Allem,Mohammed Sebahia. et al. First genetic characterization of multi-drug resistant Mycobacterium tuberculosis isolates from Algeria[J]. J Glob Antimicrob Resist,2019,14: S2213-7165(19)30120-1.

17. Vögeli B, Rosenthal RG, Stoffel GMM, et al. InhA, the enoyl-thioester reductase from Mycobacterium tuberculosis forms a covalent adduct during catalysis[J]. Journal of Biological Chemistry,2018,293(44):17200-17207.

18. Hu ZuQiong L, YanWen Z, Wen, et al. Relationship between rpoB mutations and the levels of rifampicin resistance in M.tuberculosis [J]. Chinese Journal of Zoonoses. 2016;32(01):39-44.

19. Wei HongYu L, XinYing L, Jun, et al. Genetic characteristics of isoniazid resistance of mycobacterium tuberculosis in baise, guangxi [J]. Chongqing medical. 2016;45(01):93-5.

20. Liang YaPin Z, LiLi G. Mai. Analysis of characteristics of genetic mutation of 124 cases of multidrug-resistant mycobacterium tuberculosis [J]. Journal of Clinical Pulmonary Medicine. 2016;21(04):592-4.

21. Sun $\mathrm{H}$, Zhang C, Xiang L, et al. Characterization of mutations in streptomycin-resistant Mycobacterium tuberculosis isolates in Sichuan, China and the association between Beijing-lineage and dual-mutation in gidB[J]. Tuberculosis. 2016;96(1):102-6.

22. Hassan S, Sudhakar V, Nancy Mary MB, et al. Computational approach identifies protein off-targets for Isoniazid-NAD adduct: hypothesizing a possible drug resistance mechanism in Mycobacterium tuberculosis [J]. J Biomol Struct Dyn. 2019;16(5):1-14. doi:10.1080/07391102.2019.1615987.

23. Sun J, Zhu D, Xu J, et al. Rifampin resistance and its fitness cost in Riemerella anatipestifer [J]. BMC Microbioligy. 2019;19(1):107.

24. Ramazanzadeh R, Mohammadi B, Mohajeri P. Mutations in embB gene associated with resistance to ethambutol in Mycobacterium tuberculosis strains isolated from TB patients in the west of Iran (2014-15) [J]. Int J Mycobacteriol.

Page $10 / 13$ 
2016;5(I1):140.

25. Djemal SE, Camperio C, Armas F, et al. Detection of a streptomycin-resistant Mycobacterium bovis strain through antitubercular drug susceptibility testing of Tunisian Mycobacterium tuberculosis complex isolates from cattle [J]. BMC Veterinary Research. 2018;14(1):296.

26. Ali A, Hasan Z, McNerney R, et al. Whole genome sequencing based characterization of extensively drug-resistant Mycobacterium tuberculosis isolates from Pakistan[J]. PLoS One. 2015;10(2):e117771.

27. Mekonnen D, Admassu A, Mulu W, et al. Multidrug-resistant and heteroresistant Mycobacterium tuberculosis and associated gene mutations in Ethiopia[J]. International Journal of Infectious Diseases. 2015;39(12):34-8.

28. Qin L, Huang $\mathrm{CH}$, Xu D, et al. Molecular mechanism for the activation of the anti-tuberculosis drug isoniazid by $\mathrm{Mn}(\mathrm{III})$ : First detection and unequivocal identification of the critical N-centered isoniazidyl radical and its exact Iocation[J]. Free Radic Biol Med. 2019,15: S0891-5849(19)30513-1.

29. Unissa AN, Subbian S, Hanna LE, et al. Overview on mechanisms of isoniazid action and resistance in Mycobacterium tuberculosis[J]. Infection Genetics Evolution. 2016;45(6):474-92.

30. AC N, C AN. N, et al. Performance of Genotype MTBDRplus in the Detection of Resistance to Rifampicin and Isoniazid Among Clinical Mycobacteria Isolates in Ilorin, Nigeria[J]. Curr HIV Res. 2015;13(4):308-22.

31. Khosravi AD, Sirous M, Abdi M, et al. Characterization of the most common embCAB gene mutations associated with ethambutol resistance in Mycobacterium tuberculosis isolates from Iran[J]. Infection Resistance. 2019;12(6):579-84.

32. Smittipat $\mathrm{N}$, Juthayothin T, Billamas $\mathrm{P}$, et al. Mutations in rrs, rpsL and gidB in streptomycin-resistant Mycobacterium tuberculosis isolates from Thailand[J]. Journal of Global Antimicrobial Resistance. 2016;4(3):5-10.

33. F R, A I F MH, et al. Screening for streptomycin resistance conferring mutations in Mycobacterium tuberculosis isolates from Iran[J]. J Chemother. 2016;6(5):1-5.

34. Xudong GAO, Xin HUANG, Baocheng HAO, et al. Research progress on the detection methods and drug resistance mechanisms of Mycobacterium tuberculosis[J]. Heilongjiang animal husbandry veterinary. 2016;509(17):68-72.

35. Wang Z, Xie T, Mu C, et al. Performance of Sequencing in Predicting Ofloxacin Resistance in Mycobacterium tuberculosis from Positive Bactec MGIT 960 Cultures[J]. Ann Clin Lab Sci. 2018;48(1):69-74.

36. Sirous M, Khosravi AD, Tabandeh MR, et al. Molecular detection of rifampin, isoniazid, and ofloxacin resistance in Iranian isolates of Mycobacterium tuberculosis by high-resolution melting analysis[J]. Infection Resistance. 2018;11:1819-29.

37. L Y, L M. Y L, et al. Genotypic characteristics of Mycobacterium tuberculosis circulating in Xinjiang. China[J]Infectious Disease. 2016;48(2):108-23.

38. Liu Yuan. Study on spatial aggregation of tuberculosis patients and genetic diversity of mycobacterium tuberculosis in rural areas of east China [D]. Fudan University, 2014.

39. Yang JinMin Q, Lin H, Jia. The "trinity" transformation in xinjiang and the level analysis of tuberculosis patient discovery [J]. Disease control prevention bulletin. 2016;31(01):60-1.

40. Bruni R, Taffon S, Equestre M, et al. Key Role of Sequencing to Trace Hepatitis A Viruses Circulating in Italy During a Large Multi-Country European Foodborne Outbreak in 2013[J]. PLoS One. 2016;11(2):e149642.

41. Sabir JS, Lam TT, Ahmed MM, et al. Co-circulation of three camel coronavirus species and recombination of MERSCoVs in Saudi Arabia[J]. Science. 2016;351(6268):81-4.

42. Gilchrist CA, Turner SD, Riley MF, et al. Whole-genome sequencing in outbreak analysis[J]. Clin Microbiol Rev. 2015;28(3):541-63.

\section{Figures}




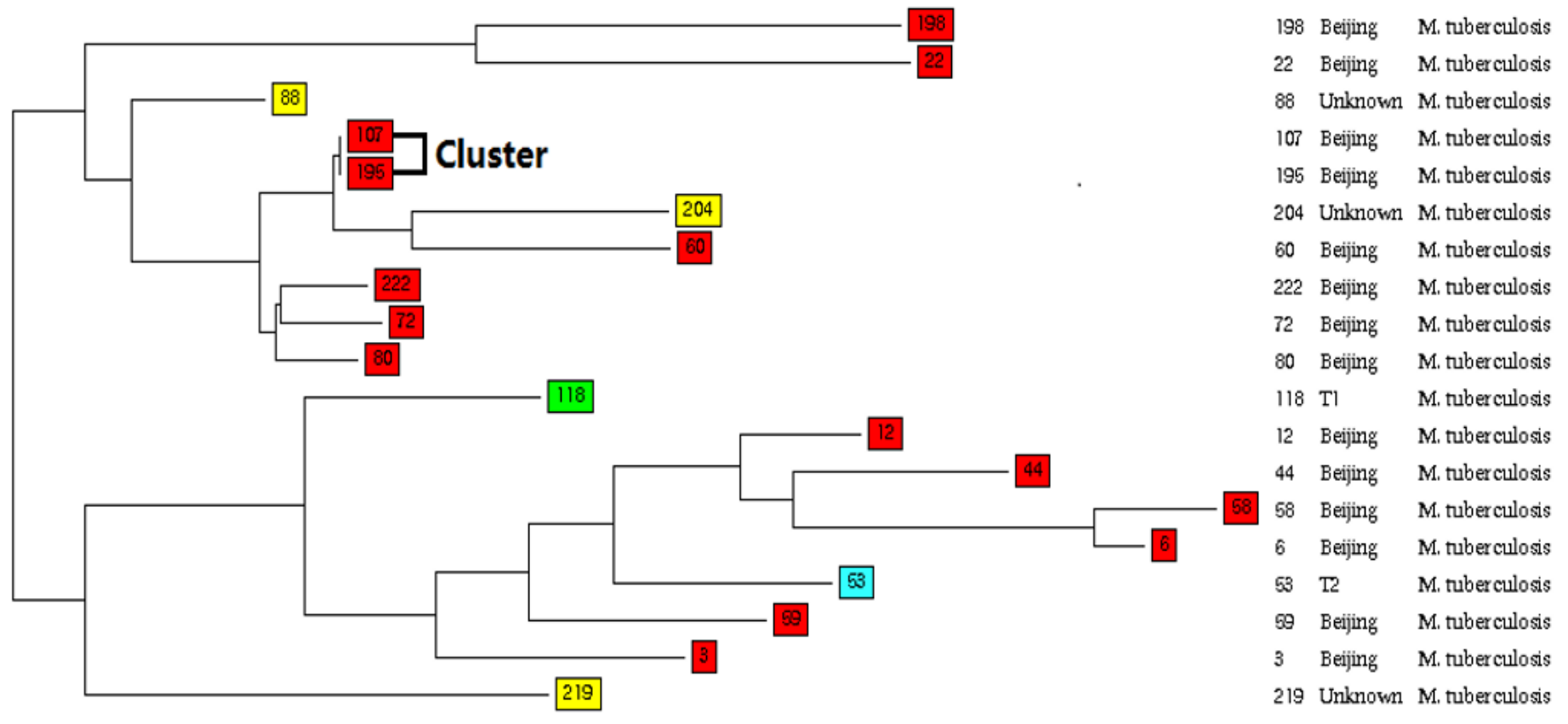

0.1

NJ-Tree, MIRU-VNTR [12]: Categorical

\section{Figure 1}

19 strains of multi-drug resistant strains of MTB gene phylogenetic tree 

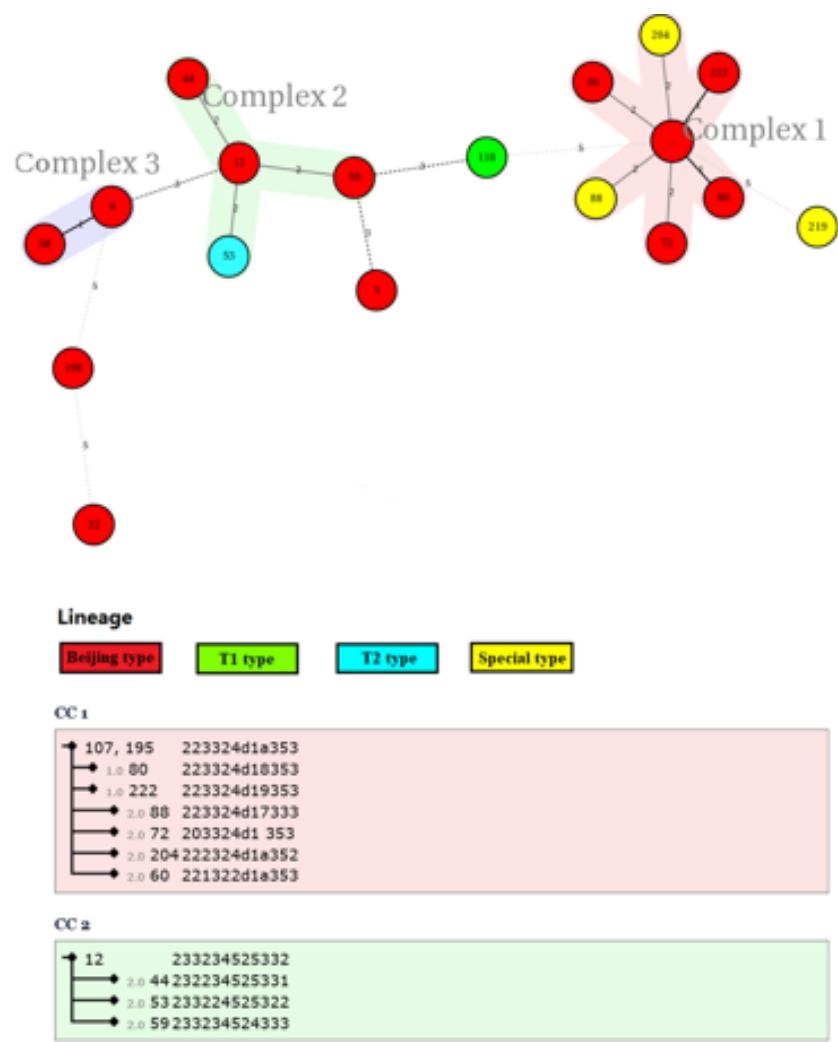

$\stackrel{\mathrm{CC}_{3}}{\mathrm{~L}^{6}} \begin{array}{r}252234525232 \\ \longrightarrow\end{array}$

Singletons

$22 \quad 243222 \mathrm{e} 18233$

118233334527353

219223335516343

$3 \quad 233424521333$
198242224017232

\section{Figure 2}

The minimum spanning tree complex paths Remark: We had analyzed the minimum spanning tree complex paths to gene type of 19 multi drug resistant Mycobacterium tuberculosis. Different color represents different gene type. The size of circle represents arise frequency of multi drug resistant Mycobacterium tuberculosis genotype. And the number on the line represent distance between difference gene type. 